\title{
La Kurzgeschichte en Alemania tras la Segunda Guerra Mundial: el caso Kaschnitz'
}

\author{
The Case of Kaschnitz \\ SANTIAGO MARTÍN ARNEDO \\ Universidad de Granada \\ España \\ smarnedo@hotmail.com
}

The Kurzgeschichte in Germany after the Second World War:

(Recibido: 26-I2-2OI7; aceptado: OI-08-20I8)

\begin{abstract}
Resumen. Me propongo en este artículo caracterizar la estructura del relato corto en la obra de la escritora alemana Marie Luise Kaschnitz (1901-1974) desde una doble perspectiva: por un lado, desde el contexto general de la literatura alemana en los años posteriores a la Segunda Guerra Mundial, destacando los puntos comunes con sus contemporáneos, y por otro lado, desde la especificidad propia de una escritora muy original que creó sus propios formatos literarios.
\end{abstract}

Palabras clave: Kaschnitz; Kurzgeschichte; Kahlschlagliteratur; Alemania; postguerra.
Abstract. This article proposes to characterize the structure of the short story in the work of the German writer Marie Luise Kaschnitz (190I-1974) from a double perspective: On the one hand, from the general context of German literature in the years after the Second World War, highlighting the common points with her contemporaries. On the other hand, from the specificity of a very original writer who created her own literary formats.

Keywords: Kaschnitz; Kurzgeschichte; Kahlschlagliteratur; Germany; postwar.

\footnotetext{
I Para citar este artículo: Martín Arnedo, Santiago (2019). La Kurzgeschichte en Alemania tras la Segunda Guerra Mundial: el caso Kaschnitz. Alabe 19. [www.revistaalabe.com] 


\section{Alemania: año cero}

La Kurzgeschichte - relato o historia corta, literalmente- tardó mucho tiempo hasta ser acuñada como un género específico (Kuipers, I970: 2). Hasta I945 prácticamente este término englobaba cualquier tipo de narración breve con diferentes características. En el siglo anterior había estado emparentada como género con las publicaciones periódicas, tales como las revistas.

El modelo estadounidense de la short story se distinguió de la long short story y encontró su reflejo en la Kurzroman o la Novelle, caracterizadas por su brevedad y por conservar la unidad de la acción (Freese, r974: 92). El maestro del relato² en estos primeros albores fue el escritor norteamericano Ernest Hemingway (I899-I96I), quien supo, gracias a su formación como periodista, sintetizar escuetamente los hechos de la vida cotidiana, pero revestidos de un gran valor metafórico y simbólico, en sus cuentos. Junto a él, William Faulkner (I897-I962), Raymond Carver (I938-I988), Edgar Allan Poe (I809-I849) -quien convirtió además los relatos en tema de discusión estética ${ }^{3}$ - o F. Scott Fitzgerald (I896-I940), se convirtieron en algunos de los precedentes ${ }^{4}$ y modelos de los escritores de historias cortas de la postguerra alemana, tales como Heinrich Böll5 (I9I7I985), Wolfgang Borchert (I92I-I947), Wolfgang Weyrauch (I904-I980) o Wolfdietrich Schnurre (I920-I989). En este primer estadio de desarrollo, el relato muestra un acontecimiento que se caracteriza, dada la cercanía del trauma bélico, por poner de manifiesto la vulnerabilidad de la existencia humana.

En el contexto posterior a la contienda, la técnica del relato ${ }^{6}$ no fue más que el esfuerzo por hacer comprensibles o asimilables las terribles vivencias de la guerra. El clima social se compone de los supervivientes que han de organizarse para retirar los escombros y volver a construir sus ciudades ex nihilo. Paralelamente, han de iniciar una nueva vida desde el punto de vista espiritual: “ein geistiger Klärungsproze $\beta$ war erforderlich”?

\footnotetext{
${ }^{2}$ Para esbozar genéricamente estos antecedentes del relato alemán sigo a Marx, L. en su obra Die deutsche Kurzgeschichte. Springer-Verlag. (2005: 90 y ss.)

3 Poe, E. A. (I846). The Philosophy of Composition. Graham 's American Monthly Magazine, vol. XXVIII, I63-I67.

4 “El short story norteamericano constituyó una revelación y el estímulo exterior para iniciar algunos ensayos en esta forma literaria" (Langenbucher, I970: 435).

5 Heinrich Böll afirma de la Kurzgeschichte: "sie bleibt für mich die reizvollste Prosaform” ["sigue siendo para mí la forma narrativa más incitante" (cit. en Bienek, I962: I70), porque trata el tema del tiempo nuclearmente, es decir, en ella cabe tanto el instante como la eternidad. Denuncia este escritor que cuando el editor encarga a un escritor un relato como si fuera un género menor, como algo que se hace sin problemas, se cae en un profundo error de concepción, "es kann Jahre dauern, ehe ich mit einer Kurzgeschichte zu Rande kommen, das heißt, ehe ich sie hinschreiben kann" ["pueden pasar años hasta poder completar un relato, es decir, hasta que puedo escribirlo"] (Schweikert, 1984: I70).

${ }^{6}$ Utilizaré como sinónimos Kurzgeschichte y Erzählung -"narración corta” y "relato"- en pro de la simplicidad y la claridad metodológica, aunque en otros contextos las traducciones puedan ser variadas (narración, historia corta, etc.). De este modo me hago eco de la indistinción que la propia Kaschnitz asume también respecto a estas palabras - a veces habla incluso de Kurze Erzählung (Schweikert, I984: 292). Estoy convencido de que esta polisemia en nada empaña la claridad y el rigor de la investigación.

6 “Era necesario un proceso de autoaclaración espiritual” (Grabert-Mulot-Nürnberger, ı982: 368).
} 
Algunos autores encaran el comienzo desde una perspectiva cristiana (por ejemplo Reinhold Schneider en su drama de $195^{2}$ Innozenz und Franziskus), y otros, nihilista (sirva como ejemplo la pieza teatral Draußen vor der Tür de Wolfgang Borchert, estrenada en 1947). En cualquier caso, la herida social es profunda y provoca incluso disputas entre los intelectuales, entre aquellos que se fueron (v.g. Thomas Mann) y los que, quedándose, optaron por el "exilio interior". Thomas Mann aireó duramente esta controversia con estas palabras publicadas en el Augsburger Anzeiger:

Es mag Aberglaube sein, aber in meinen Augen sind Bücher, die von I933-1945 in

Deutschland überhaupt gedruckt werden konnten, weniger als wertlos und nicht gut in die Hand zu nehmen. Ein Geruch von Blut und Schande haftet ihnen an. Sie sollten alle eingestampft werden. ${ }^{8}$

Más allá de la brecha ideológica que se abre entre los que se fueron y los que se quedaron, para los escritores del momento, el relato como forma literaria tenía muchos atractivos. Lo que caracterizó al relato en esta fase inicial fue la descripción de un acontecimiento singular. Acontecimiento que irrumpe dentro de la normalidad de forma imprevista. De los personajes no se suele conocer el nombre, para que el lector pueda identificarse con la situación sin que le parezca algo lejano o ajeno -de hecho, en los relatos de Kaschnitz como Das Dicke Kind (1952), Der Bergrutsch (1952) o Die Pilzsucher (1948-I955?), entre muchos otros, los protagonistas aparecen innombrados-. El estilo, llano y sin ornamentos, contribuye a esta atmósfera de normalidad que será violentada en cierto momento -por ejemplo, en Der Strhohhalm (I96o) o en Eisbären (I966), el lenguaje es extremadamente sencillo-. El final suele presentarse sorpresivamente y propone un giro argumental -y aquí prácticamente todos los relatos de Kaschnitz valdrían de ejemplo. Este final acostumbra a erigirse en momento álgido de la narración y obliga al lector a repensar toda la historia de nuevo, incluso a una relectura. Que además quede abierto es otra estrategia narrativa para propiciar el trabajo de co-creación por parte del lector. Todas estas características encajan perfectamente en la mayoría de los relatos de Kaschnitz.

De modo que la implantación de este género de lectura rápida tuvo mucho que ver en Alemania con circunstancias socio-históricas. En primer lugar, la gente, por un lado, experimentó cómo dentro de sus vidas cotidianas irrumpían acontecimientos de naturaleza traumática -detenciones, cadáveres, etc.- y esto influyó en la concepción de la Kurzgeschichte como relatos de lo inesperado. La sensación de verse desbordado por las circunstancias advenidas se compadece con una visión de la vida como algo que puede sorprender brusca y desagradablemente en cualquier momento. Este rasgo es esencial: saber que somos vulnerables, y que en cualquier momento la vida nos puede dejar debilitados.

\footnotetext{
8 "Quizá sea superstición, pero en mi opinión, aquellos libros que pudieron ser impresos en Alemania entre 1933 y 1945 tienen menos valor que lo despreciable y no son agradables a la vista. Un rastro de sangre y de vergüenza permanece en ellos. Todos estos libros deberían ser destruidos" Grabert-Mulot-Nürnberger (ig82: 368 ).
} 
El segundo rasgo, junto al de vulnerabilidad, es el de la sencillez de la expresión-y por tanto su fácil acceso a todo tipo de lector-, que tiene que ver con la sensación de nuevo inicio. Y no sólo con la sensación, sino también con la necesidad de un inicio absoluto, de un punto cero, de comenzar la historia de nuevo, y los autores alemanes con ayuda de textos sencillos y breves se propusieron distanciarse de las obras ideologizadas y artificiosas de su pasado inmediato. Ha de comenzarse por lo sencillo. Además, así podían conectar mejor con el proceso de la población. Como señala Wolfgang Weyrauch: "wo der Anfang der Existenz ist, ist auch der Anfang der Literatur"9. Este autor publicó en I949 una influyente antología de Kurzgeschichten que recoge obras de Luise Rinser, Herbert Roch -cuyo relato da título a la antología-, Bruno Hampel y otros autores entre los que no está Kaschnitz. Lo más relevante de esta publicación es que se convirtió en una especie de manifiesto de lo que se ha dado en llamar la Kahlschlagliteratur o Literatur der Stunde Null -“literatura del punto cero"-, es decir, el movimiento al que se adscriben en su momento autores como Günter Grass, Uwe Johnson o Arno Schmidt, y que alude precisamente al comienzo de una nueva era de expresión literaria en 1945 (Schnell, 200I: 479-5 IO).

Kaschnitz había pertenecido también a la Trümmerliteratur-la literatura que nace como respuesta a, y a partir de, los escombros de la guerra y que temáticamente y cronológicamente está muy emparentada con la Kahlschlagliteratur- y hay que hacer notar que Kaschnitz tras la guerra se va decantando cada vez más por el género del relato, hasta el punto de que prácticamente todos los relatos que publica lo hace en su etapa de madurez: en I96o Lange Schatten y en i966 Ferngespräche. Sin embargo no fue pionera en el género en Alemania (Pulver, 1984: 4I). El precursor había sido Wolfgang Borchert. Hasta comienzos de los cincuenta, que es cuando Kaschnitz publica Das dicke Kind, Elisabeth Langgässer había publicado ya su ciclo Torso en I947, y en I949, el volumen ya aludido de Weyrauch.

En estos movimientos literarios, los recursos son mínimos, porque la construcción del futuro debe empezar sin presupuestos, en paralelismo a la reconstrucción de un país en ruinas. Cierto es que Weyrauch incluyó en la antología narraciones modélicas de los dos siglos anteriores -Chéjov, Heinrich von Kleist o Guy de Maupassant- donde se muestran los lazos comunes con la tradición y las diferencias, sobre todo temáticas (soldados en el frente, campos de concentración), de ahí que Weyrauch indique que hay dos tipos de literatura: la que tiende a la perfección estética y la experimental que se abre al futuro. Los autores se encuentran en una encrucijada y entre ambos extremos han de buscar su lugar. Desde el principio se encasilló a Marie Luise Kaschnitz en el lado de los tradicionalistas. Este trabajo pretende mostrar, entre otras cosas, la inexactitud de tal adscripción.

9 "Allí donde comienza la existencia, comienza también la literatura” (Weyrauch, I949: 2I7). 
En los años cincuenta hubo otra oleada de publicaciones de relatos, y en los setenta se hicieron muy populares los concursos literarios. En la actualidad, el relato se ha reivindicado como un género no menor, puesto que las condiciones aceleradas de la vida actual no facilitan lecturas de grandes trazos y el formato de lo breve y veloz se va instalando cada vez más en nuestras costumbres. Pero estas razones de aceleración social actual son diferentes a las causas que motivaron la difusión del género en tiempo de Kaschnitz.

Hay cierto acuerdo entre los estudiosos ${ }^{10}$ en la enumeración de ciertas características comunes en las Kurzgeschichten, de las cuales nosotros vamos a destacar nueve que se hallan presentes especialmente en los relatos de Kaschnitz:

- El comienzo se introduce de inmediato en la acción o en la atmósfera, sin preámbulos ni aclaraciones: "Kaschnitz ist eine Virtuosin des ersten Satzes" ["una virtuosa de la primera frase"] (Bönnighausen, 20I3: 444). Los antecedentes de los personajes se aclaran parcialmente a lo largo de la lectura o bien han de ser colegidos entre líneas. Con esto el lector se siente atrapado por el texto y la tensión está asegurada, ya que siempre se quiere saber más. Como ejemplos seleccionados que tienen tal característica basten Judith. Ein Gespräch (1946), Die Abreise (1950), Das dicke Kind (1952) o Am Circeo (196o).

- El final permanece abierto o semiabierto. La historia simplemente deja de ser contada y muchas preguntas planteadas durante el relato no han sido respondidas. Esto obliga al lector a trabajar en la confección de la historia y a seguir haciéndose preguntas sobre la misma. Con lo cual la influencia de la narración no acaba donde termina el relato, sino que continúa en la cabeza del lector. Esta característica es muy usada por la autora, valgan sólo como ejemplo Der Strohhalm (1960), Das dicke Kind (1952), Eisbären (1966), Vogelrock (1966) o Gespenster (I96o).

- La acción que se desarrolla en el relato pertenece a la vida cotidiana y cada lector se puede identificar fácilmente con lo que lee, aunque quizá se trate de una cotidianidad engañosa. Pueden aparecer conflictos, reinterpretaciones, giros argumentales, pero el marco argumental nunca es extraordinario o fantasioso. Ejemplos: Die Schlafwandlerin (1952), Der Bergrutsch (1952), Jennifers Träume (1966) o Schiffsgeschichte (1966).

- Los personajes son asimismo personas normales, que no sobresalen por ninguna característica. A menudo al ser simplemente esbozados, se impregnan de cierto misterio. Ejemplos: Der Gärtner (1948-1955?), Der Spaziergang (19271931?), Das Leben nach dem Tode (1926), Wege (1960) o Nesemann (1952).

\footnotetext{
${ }^{10}$ Doderer, K. (1977). Die Kurzgeschichte in Deutschland. Ihre Form und ihre Entwicklung. Wiesbaden: Metopen. Durzak, M. (1980). Die deutsche Kurzgeschichte der Gegenwart. Stuttgart: Reclam. Gelfert, H. (1993) Wie interpretiert man eine Novelle und eine Kurzgeschichte? Stuttgart: Reclam. Hischenauer, R. Weber, A. (edit) (1974). Interpretationen zu Marie Luise Kaschnitz. Erzählungen. München: Oldenburg Verlag.
} 
- El tiempo en que transcurre la acción suele ser breve, de forma que el tiempo de lectura se acerca mucho al tiempo narrativo, tendiendo a la coincidencia. Si bien a veces ciertas intrusiones y divagaciones psicologistas así como descripciones, suelen dilatar el tiempo narrativo. Eisbären (I966), transcurre en una noche, Das dicke Kind (1952), en una tarde, Der Spaziergang (1927-I931?), en una tarde o Ein Mann, eines Tages (I966) es tan sólo un rato, como Vogelrock (I966).

- Casi siempre la historia es contada desde un yo-narrativo, en singular y suele coincidir el narrador con el protagonista. Prácticamente todos los relatos son en primera persona, y con muy pocas excepciones -Wer kennt seinen Vater (I966), Der Spinner (I966) o Der Tunsch (I966)-, son femeninos: Eisbären (ı966); Ja, mein Engel (1966); Lupinen (I966), Das dicke Kind (1952) o Am Circeo (г96о).

- Se crea una especial complicidad entre narrador y lector, hasta el punto que el primero no sabe responder ni solucionar los interrogantes que se plantean en el decurso de la historia. La mezcla de apertura y clausura fuerzan al lector a implicarse en la trama y a identificarse con el personaje ${ }^{\text {I: }}$ Wege (I96o), Das fremde Land (ı96о), Der Spinner (ı966), Das Wunder (ı96о) o Rätsel Mensch (1948-1955?).

- Se plantea un acontecimiento extraordinario, que sin previo aviso se desprende de lo ordinario, lo que fue acuñado con el término Schicksalsbruch. Nada vuelve a ser igual después de este acontecimiento: Vogelrock (I966), Martin we want a lesson (1966), Schiffsgeschichte (1966), Die Füße im Feuer (1966) о Das dicke Kind (1952).

- El lenguaje tiende a ser sencillo, sin grandes complicaciones o figuras literarias. En este sentido pretende acercarse a la cotidianidad lo más posible: Pax (1952), Märzwind (1952), Das ewige Licht (1960), April (1966), Der Schriftsteller (I966).

Las características arriba enumeradas no son por supuesto suficientes ni necesarias, pues una hermenéutica, por muy exhaustiva que se proponga, jamás puede agotar el sentido ni la configuración de su objeto. Aún así, suponen un marco teórico muy solvente para el interés analítico del estudioso y abren caminos de interpretación que tendrán que mostrar su fecundidad.

\footnotetext{
II Titzmann (1977: 247).
} 


\section{Los relatos de Kaschnitz: visión sinóptica}

Pese a no haber sido reconocida primordialmente como autora de relatos, se tiene noticia de que Kaschnitz llegó a escribir Io6 relatos. 7o fueron publicados en vida, de los 36 restantes, 26 han aparecido en las Obras Completas, que aunque fueron descartados por incorrecciones e imperfecciones para su publicación, constituyen, a juicio de su autora, una buena fuente de conocimiento de su labor productiva. Quedan Io relatos que no aparecen en las Obras Completas, porque fueron rechazados frontalmente por la autora y tal decisión fue respetada por los editores.

Respecto a los 7o relatos publicados, básicamente su aparición la distribuiremos en tres etapas, según una división que la misma autora aceptó. Incluiremos algunos datos más para contextualizar los relatos:

A) I933-I944. Aparecen sus primeras novelas: Liebe beginnt (1933) y Elissa (1937). Los rasgos autobiográficos empiezan a jugar un papel importante, pero de manera muy indirecta, sobre todo en Liebe beginnt, donde novela el encuentro con su futuro marido Guido von Kaschnitz.

Publica cuatro relatos: Der Geiger (г9I9), Spätes Urteil(1930), Dämmerung (I930) y Das Wiedersehen (r933). Estos primeros relatos son de tentativa de estilo y de hacerse con un camino propio. La reflexión estética tiene importancia manifiesta en el labrarse un camino propio como escritora.

B) I945-I957. La autora experimenta un viraje en su estilo y concepción de la creación literaria, sobre todo a partir de las biografías que escribió: Roman eines Malerlebens (1949), que más tarde aparecería con el título en I967 de Die Wahrheit, nicht der Traum, Florens (sobre la juventud de Joseph von Eichendorff, a partir de sus diarios y vivencias) y una biografía, si bien con menos artificio literario y más centrada en un sobrio informe cronológico, sobre su marido. El yo experimenta una inflación y se hace omnipresente. Y comienza a publicar ensayos, como Menschen und Dinge (1946) en los que la realidad es analizada en función de esa perspectiva. Aparece la así llamada "prosa autobiográfica" Engelsbrücke (1955) donde se describe sobre todo su estancia en Roma, y Das Haus der Kindheit (1956).

Cultiva con más dedicación el relato, y aparte de publicar Judith (1946), Die Abreise (1950) y Das Fräuchen (1952), publica una colección de relatos Das dicke Kind (I952) que contiene el relato que da título a la colección y que se convertiría en uno de los más famosos y analizados. Este primer libro contiene relatos tan importantes como Die Schlafwandlerin -que trata el tema del doble-, Nesemann-que se ocupa del mundo de la infancia con tintes autobiográficos- y Der Bergrutsch -que trata enigmáticamente el tema del destino y de la muerte. Prácticamente los temas y las formas del relato aparecen ya en este primer libro, aunque en su etapa de madurez, la indagación psicológica se radicaliza.

C) 1958-1974. El hecho trascendental es la muerte de su marido (analizado por ejemplo en Wohin denn Ich en I963) y la concentración radical en el análisis del yo. En su prosa autobiográfica el yo se hace presente cada vez más explícitamente. Publica Beschreibung eines Dorfes y Tage, Tage, Jahre (Aufzeichnungen). 
Es la época de madurez, cuando la escritora comienza verdaderamente a navegar contracorriente. A finales de los años cincuenta la literatura en Alemania se vuelve hacia un compromiso social -Verteidigung der Wölfe gegen die Lämmer de M. Enzensberger en I957-, la actualidad política ocupa un puesto central -Mutmassungen über Jakob de Uwe Johnson en I959-, incluso el pasado nazi-Die Blechtrommel de G. Grass-. Y sin embargo Kaschnitz se vuelve hacia sí misma, influida por la circunstancia biográfica de la muerte de su marido (I958), que no sólo le cambia la vida y la misma actividad práctica de escribir (Pulver, r984: 75), sino que le da un cariz de interiorización a su obra de la que ya no se desprenderá nunca.

La segunda decisión que le hace tomar un camino propio más allá de las modas de su tiempo fue una radicalización del enclaustramiento -y no sólo en un sentido políticodel yo en sí mismo. En Menschen und Dinge (I946), el yo estaba presente, pero como una parte más del mundo. Ahora se trata de un yo distanciado del mundo, exterior a él. Baste citar dos títulos paradigmáticos de esta etapa, para comprobar que el yo se hace presente en el mismo título Dein Schweigen-meine Stimme (1962) y Wohin denn ich (1963).

En esta tercera etapa aparece el grueso de sus relatos. Su actividad se incrementa y en apenas un lustro publica más de cuarenta relatos. Además de Das Mädchen von $S$. Agata (1958), Wenn's wieder geschähe (1958), Alle Jahre wieder (1962), Martin, we want a lesson (I966), Der Statthalter (I966), Was war das für ein Fest (I966) y Das Märchen vom Machandelboom (1974), aparecen las colecciones Lange Schatten (1960), Ferngespräche (I966) y Vogel Rock (I969). Aparte de otra recopilación (Eisbären) que no incluye relatos nuevos.

En Lange Schatten (1960) hay que destacar, además del relato que da su título al del libro -que habla sobre la adolescencia en un contexto veraniego-, Der Strohhalm-un agudo retrato psicológico sobre la inseguridades de una mujer, Das Wunder-ambientado en la Navidad- o Schneeschmelze -convertido luego en un exitoso Hörspiel, y donde se relata los sentimientos de miedo de un matrimonio mayor ante la posible llegada de un hijo adoptivo ya fallecido.

Ferngespräche (I966) es el tercer libro de relatos. Publicados con muy poco tiempo de separación, apenas unos pocos años, no hay como conjunto diferencia sustancial de evolución, más bien, cada relato es único, aparte de incorporar relatos publicados. Sí se percibe una radicalización de las situaciones: por ejemplo en Die Füße im Feuer describe con mucha dureza la descomposición moral de una mujer hasta arrastrarla a la locura; $J a$, mein Engel, también roza los bordes de la locura, en una retrospectiva relatada desde la tumba. Ferngespräche, por contra, mantiene un tono más distendido para criticar la falta de compromiso y superficialidad en las relaciones sentimentales de la modernidad. Otros núcleos temáticos son el nazismo -Ein Tamburin, ein Pferd o Lupinen-, lo sobrenatural -Eisbären o Schiffsgeschichte- o la imposibilidad de asimilar la propia autobiografía -Zu irgendeiner Zeit o Ein Mann, eines Tages-.

Vogel Rock (I969) incluye únicamente seis historias, y la mayoría ya publicadas, en las que hay que destacar Der Spinner, -las rarezas de un trabajador de la Oficina de 
Tráfico que desemboca en paranoia, sin que el lector sepa señalar el momento en el que se traspasa la línea de la enfermedad mental- y Jennifers Träume -un relato lleno de fantasía, en la que una niña mezcla en sus sueños realidades paralelas.

Al final de su vida, sin que constituya propiamente una cuarta etapa, hay que destacar su ciclo poético Ein Wort weiter. Ahí pretende responder a su libro anterior, Wohin denn ich, señalando el camino del lenguaje, para superar el sufrimiento, los límites y aún el mismo yo. El lenguaje se purifica aún más. Ya no necesita en sus poemas efectos rítmicos, pictóricos o de otra índole. La palabra se reduce a su mínima extensión para exprimir su máxima intensión.

\section{Rasgos genéricos. Apreciaciones de Kaschnitz}

Kaschnitz no se interesó especialmente en teorizar sobre la Kurzgeschichte, y por comodidad, según la opinión de Bachmann-Schweikert ${ }^{12}$, habría utilizado el término más amplio de Erzählung. En realidad la artista no necesita ni está obligada a reflexionar teóricamente sobre su producción. De hecho, cuando se le encomienda el seminario de verano de la Universidad de Fráncfort sobre las grandes figuras de la literatura europea, Kaschnitz rehúye cualquier tipo de magisterio: "ich habe im Frankfurter Seminar keine Gedichte analysiert. Ich habe die Teilnehmer gebeten, selbst etwas zu schreiben, um sich mit den Problemen des Ausdrucks und der Form vertraut zu machen”³. Algunos teóricos de la estética incluso han considerado incompatible la creación con la reflexión sobre los medios formales ${ }^{\mathrm{I}}$.

Aun así, circunstancialmente se vio obligada a reflexionar sobre los medios formales que utiliza la Kurzgeschichte. Una de esas ocasiones fue en r95o. La Sociedad "Für die Jugend-Pro Juventute” organizó en Maguncia un monográfico sobre la Kurzgeschichte e invitó a Kaschnitz. La lección fue publicada por primera vez en el séptimo volumen de sus Obras Completas. Allí relaciona Kaschnitz la Kurzgeschichte con las Novellen de la literatura clásica universal. Entre ambos géneros no es fácil trazar una distinción, y quizá lo que más los emparenta sea la oposición respecto a la novela, como género de cierta extensión.

Al género breve -prosigue la autora- se le exige unidad de la acción, frente a la pluralidad de hilos argumentales que cruzan la novela: un lugar, un tiempo, un protagonista. Todo tiende a la singularidad. Y sin embargo, la pretensión de esta particularidad es incluir el todo (“In solcher Bechränkung gilt es dennoch, das Ganze zu fassen”)" la experiencia estética es completa y no se echa en falta nada. De hecho, lo que caracteriza

\footnotetext{
${ }^{\mathrm{I}}$ Kaschnitz (2OO2: 2O2).

I3 "En el seminario de Fráncfort no he analizado ninguna poesía. Pedí a los participantes que escribieran ellos mismos algo para familiarizarse así con los problemas de la expresión y de la forma" (Schweikert, I984: I90).

I4 Tapies, A. (1978). El arte contra la estética. Barcelona: Ariel.

I5 “Dentro de tal limitación sin embargo, se puede concebir la totalidad” (Kaschnitz, I98I vol. 7: 59I).
} 
al relato, no es tanto una serie de marcas externas o visibles -como v.g. cierta extensión-, cuanto internas -la unidad de sentido-. No es por tanto que el relato, como Poe apuntara ya en ${ }^{8} 46^{16}$, se pueda leer de una sentada (“"at one sitting”), pues esta característica es demasiado superficial.

¿Cuál es la nota definitoria y esencial de la Kurzgeschichte? El mostrar, y de este modo superar una "discontinuidad" existencial. Dicha discontinuidad consiste en la falta de relación con otro contexto más amplio. Cualquier singularidad requiere una explicación relacional. Nada se explica por sí mismo. Siempre se recurre a una causa anterior, y así en una serie de causas que se pierde en el infinito. Para Hegel, como es bien sabido, solamente podemos explicar con entera satisfacción un evento cuando se lo relaciona con el todo ${ }^{17}$. Pues si nos detenemos en un punto determinado, siempre quedará incompleto el recorrido por la cadena de causas. Y sin embargo, el todo está ya presente en cada parte. Esto es lo paradójico. Por tanto, el relato no comprueba la limitación de espacio, tiempo, lugar, etc. como algo negativo, sino más bien como una especie de minúsculo espejo en el que puede reflejar el universo entero, "ein Mikrokosmos, in dem der Herzschlag des Ganzen zu spüren ist" ${ }^{\text {"18. }}$. Pero si este pensamiento juega en Hegel un papel de reflexión muy abstracta y de muy altos vuelos, en Kaschnitz se presenta como una intuición sensible y existencial, donde la misma realidad duele y ofrece resistencia. La escritora a cambio refleja con un lenguaje muy minucioso cada detalle y singularidad de su realidad vital. Rastrea, por así decir, lo infinito en lo finito. Por eso la labor literaria tenía por fuerza que ser para ella biográfica, pues la visión está asumida siempre en primera persona.

¿De dónde surge esta necesidad de explicación? Esta es la pregunta no respondida que como un motor subterráneo pone en marcha toda su actividad creativa. Baste ahora citar las palabras de la autora: "Alle meine Figuren [...] stehen (alle) unter der Einwirkung rationalistisch nicht zu erklärender Mächte, gegen die sie ankämpfen oder denen sie sich beugen oder an denen sie zugrunde gehen" ${ }^{19}$. La falta de autofundamentación ontológica -que la aleja del optimismo racionalista de Hegel, y la sitúa más cerca del Romanticismo que explora la realidad con otros medios (sentimiento, intuición)- la expresa Kaschnitz de manera intuitiva y gráfica: “der rätselhafte Hintergrund allen Geschehens”2o.

Esta necesidad histórica de explicarse el todo no es novedosa, lo que es novedoso es la manera de plasmarla:

\footnotetext{
I6 Poe (I846: I63)

I7 Hegel afirmaba: "Das Wahre ist das Ganze" ["La verdad es el todo"]- (Hegel, r973: 24), en una concepción holista de la verdad, en la que el concepto de infinito tiene prevalencia explicativa sobre lo finito, muy acorde con el espíritu romántico y racionalista de la época.

I8 “Un microcosmos en el que se puede sentir el latido del todo". (Kaschnitz, I98I vol. 7: 592).

I9 “Todos mis personajes [...] se encuentran bajo el influjo de poder que no se pueden aclarar por medio racionales ante los cuales se inclinan o bien se precipitan por su abismo". Schweikert (I984:292).

20 "El enigmático fondo de todo acontecer”. Kaschnitz, M. L., “Die Schwierigkeit, unerbittlich zu sein. Interview mit sich selbst." En Schweikert (I984: 297-300).
} 
Los medios formales para conseguir este objetivo primordial son variados: "Die Möglichkeiten der Kurzgeschichte sind fast unbegrenzt" ${ }^{21}$. A veces no existe cénit dramático y se presenta un trozo de realidad sin "picos”, por así decir, en los que el Paukenschlag-el golpe de timbal- es casi inaudible. Otras veces no existe unidad espaciotemporal, y la misma autora propone un despliegue temporal más extenso: el relato $J a$, mein Engel (I966) es un buen ejemplo, los escenarios físicos varían (la casa, el hospital, el cementerio) y el aliento temporal es de largo recorrido (la joven hospedada pasa de ser estudiante a mujer casada y madre). La ubicación espacial, por ejemplo, varía mucho. Kaschnitz reconoce ${ }^{22}$ que cuando la acción transcurre en el extranjero, los lugares están muy especificados: verbigracia Roma o Cabo de Circe. Cuando la acción por el contrario transcurre en Alemania, la autora se limita a sugerir una calle, un jardín, etc. Lugares cotidianos pero no imprecisos: "Trotzdem habe ich auch dort [...] sehr genaue Vorstellungen” ${ }_{23}$. En el relato Wer kennt seinen Vater (I966), por ejemplo reconoce que el espacio físico ha quedado muy oscurecido, pero casi siempre las ubicaciones tienen un carácter autobiográfico: por ejemplo el Potsdam de su infancia en Das dicke Kind (1952).

Pero es sobre todo respecto al contenido de las historias donde la escritora se muestra única. La tensión narrativa está garantizada por una atmósfera de inquietud que ha sido estudiada en relación con el fenómeno de lo Unheimliche (Martín Arnedo, 20I5: 86). En efecto, la introducción de elementos mágicos, irracionales e inexplicables tienen que ver con la convicción básica, que hemos expuesto más arriba, de que la razón no puede fundamentar el orden de la existencia. Y ese fondo de abismo se traduce para sus personajes en angustia y en miedo, pero no en el sentido comercial de suspense -puesto que los finales quedan abiertos-, sino de desazón existencial. Ella ha reconocido el miedo como experiencia básica. Y su forma de combatirlo ha sido a través de la expresión literaria: "Wer ausspricht, bannt, und der Wunsch, das Schreckliche zu bannen, mag die Ursache meiner traurigen Gedichte und pessimistischen Geschichten gewesen sein” ${ }^{24}$.

Podemos afirmar que cuando Kaschnitz trata de explicitar las características de la Kurzgeschichte, algo que no acomete de buen grado, se fija más en características internas de sentido, como la completitud de la experiencia estética, que en características internas tradicionales, tales como la unidad de acción, de lugar, etc. que en ella muchas veces no se cumplen.

Hasta aquí la perspectiva interna sobre su labor creativa. En el próximo apartado, vamos a complementar esta caracterización con una perspectiva externa.

\footnotetext{
2I "Las posibilidades del relato son casi ilimitadas" (Kaschnitz, I98I vol. 7: 774).

22 (Kaschnitz, I98I vol. 3: II8).

23 “Y sin embargo tengo allí también [...] representaciones muy precisas". (Kaschnitz, i98I vol. 3: II8).

24 "Aquel que expresa, ahuyenta, y el deseo de ahuyentar lo terrible parece haber sido el origen de mis tristes poemas y de mis historias pesimistas" (Kaschnitz, I98I vol. 3: 434).
} 


\section{Tres caracterizaciones externas}

Vamos a aludir ahora a las características definitorias que otros estudiosos han encontrado en los relatos de Kaschnitz.

Examinaremos en primer lugar la aportación de la prestigiosa estudiosa de su obra, E. Pulver, quien hace hincapié en dos rasgos esenciales de los relatos: por una parte, la aparición de un punto de ruptura irreversible en la vida del protagonista y, por otra, el contraste entre la situación surrealista y el lenguaje realista que la describe. Estos dos elementos inducen a crear una atmósfera de inquietud, según hemos expuesto en el apartado anterior. A continuación analizaremos la opinión de un escritor, Siegfried Lenz ${ }^{25}$, para quien lo importante es el alumbramiento de un destino; y de un crítico, Marcel Reich-Reinicki ${ }^{26}$, quien subraya la fatalidad del que no controla su futuro.

Pulver (I984: 50) considera como texto fundacional de las narraciones de Kaschnitz su relato Das dicke Kind (1952). En cierto modo es verdad, pues en tal relato Kaschnitz acierta con una estructura narrativa que luego aplicará a otras narraciones; pero no podemos coincidir del todo con Pulver, cuando considera este relato como un mero antecedente, como un simple punto de arranque. Este relato, pese a la época temprana en que se gesta, posee la suficiente entidad y densidad como para no desdecir en comparación con sus obras de madurez. Además Kaschnitz lo publica una y otra vez, signo de que se siente ligada a Das dicke Kind en tiempos ulteriores, amén de considerarlo como el mejor texto de su primera época. Y esa feliz estructura se caracteriza a juicio de Pulver por dos rasgos:

- La aparición de un elemento catalizador, que Brustmeier (I966: 5) denominó Lebensbruch o Schicksalsbruch-ruptura de la vida o del destino- y que articula toda la tensión narrativa.

- El estilo realista del lenguaje en una situación que chirría con el sentido común. Por ejemplo, en Das dicke Kind, la niña aparece vestida de otra época, la protagonista y la niña recorren un lago que ya no existe, pero de todo esto se informa con una neutralidad en la que nadie, incluido el lector, parece sorprenderse. Se apunta de esta manera a un nivel de irracionalidad subterráneo que palpita tras la normalidad, como dice Pulver"27, aunque el lector se siente "zwar verunsichert, aber nicht ratlos”. Es un presentir, un intuir lo que siente el lector, sin llegar a verdadero saber. Solamente por medio del elemento catalizador -la ruptura del destino- el protagonista se conducirá hacia el verdadero saber, y con ello al lector, como si se tratara de una novela de formación en formato pequeño.

\footnotetext{
${ }^{25}$ Lenz, S. (I966, 2I de noviembre). "Personen mit Schicksal”. Der Spiegel.

${ }^{26}$ Reich-Renicki, M. (I966, 23 de septiembre). “... die uns überleben werden”. Die Zeit.

${ }^{27}$ Pulver (I984:50). "Inseguro, pero no perdido“.
} 
Otros intérpretes, aparte de Pulver, han hecho del destino sin embargo el eje principal desde el que articular la comprensión de los relatos de Kaschnitz. Una especie de predestinación que determina el camino de la vida. Siegfried Lenz en su crítica del libro Ferngespräche (ı66: ı63), opina que, salvo en algunas excepciones - Wer kennt seinen Vater o Zu irgendeiner Zeit- el destino no es propiamente nombrado, mas sí cumple una función determinante. Los personajes estarían condicionados por un destino no evidente para ellos. Se percatan de él de una forma velada y tácita, y se produce lo que él llama una Augenöffnung, un darse cuenta, un des-cubrir. Son personajes que por así decir deben atravesar su propio vía crucis. En palabras de Lenz, Kaschnitz: "ist ein Plädoyer für die Anwesenheit von Schmerz, Trauer und Tragik in der Welt” ${ }^{28}$. Y la resolución del conflicto viene codificada a través de una palabra-llave, la mayoría de las veces enunciada en el título: v.g. Eisbären, Die Pflanzmaschine o Das dicke Kind.

Para justificar su propuesta, Lenz propone analizar las historias desde el punto del vista del destino: por ejemplo, en el relato Lupinen, la mujer judía que acaba suicidándose tras haber perdido a su hermana en su común huida en el tren que las transportaba al campo de concentración, o también en Eisbären, en donde aparece un matrimonio herido por la ocultación de un viejo amor. El pasado, como en las tragedias griegas, no deja más que un camino posible por recorrer.

Todas estas historias son, para Lenz, una mirada introspectiva a la subjetividad, son el resultado de, según su peculiar expresión: "wenn wir uns mit uns selbst verabreden". Por tanto el relato nos induce a caminar hacia la interioridad con una intención muy cercana al psicoanálisis: sólo alumbrando lo que ha permanecido oculto posibilita la superación de la represión que supone seguir manteniéndolo oculto.

También el prestigioso y ya fallecido crítico literario Marcel Reich-Ranicki adopta una óptica parecida. Asegura que los relatos de Kaschnitz son "Geschichten, deren Atmosphäre in der deutschen Gegenwartsliteratur einmalig ist. Einige dieser Geschichten werden uns überleben" ${ }^{\circ}$. Y después procede a analizarlos como ejemplos de cómo los personajes están lastrados por sus destinos. Por ejemplo, en Die chinesische Cinelle, el percusionista que falla el golpe de gong en un momento crucial de un concierto, fracasa de por vida en los momentos cruciales que se le plantean en el trabajo, en las relaciones, etc. El recluso de Die Pflanzmaschine tras volver a casa y encontrar a su mujer con otro, voluntariamente se vuelve a prisión.

Aunque el análisis de Pulver y la caracterización de lo "inquietante” como categoría válida de acceso al análisis de la estructura de los relatos es solvente, la óptica de Lenz o de Reich-Ranicki es también legítima y parece fructífero adoptar la perspectiva sobre Kaschnitz como una fatalista que contempla con pesimismo el decurso de la vida: cómo en

\footnotetext{
28 “Presenta un informe sobre la presencia del dolor en el mundo, del duelo, y de la tragedia” Lenz (ı966: ı64).

29 “Como cuando quedamos con nosotros mismos” Lenz (ı966: ı64).

30 "Historias, cuya atmósfera es única en la historia de la literatura alemana contemporánea. Algunas de estas historias nos sobrevivirán" (Reich-Ranicki, I966).
} 
unas pocas páginas se rompe el destino, la irrupción de la predestinación y la irracionalidad de las fuerzas que mueven a los personajes lo justifican.

\section{Conclusiones}

Kaschnitz comparte con su generación ciertas notas comunes en su quehacer literario respecto a los relatos. Dada la proximidad de la experiencia de la Segunda Guerra Mundial dichos relatos, que sobrepasaron el centenar, están marcados por una conciencia omnipresente de la fragilidad de la vida, por la irrupción de circunstancias extremas y la imposibilidad de zafarse de ellas.

En este sentido, forma parte de la historia del género alemán Kurzgeschichte y encuentra su lugar-atendiendo al primer objetivo de situarla en el contexto general de la literatura alemana de postguerra- en el movimiento llamado "literatura de punto cero", junto a autores como Uwe Johnson o Heinrich Böll. Intentan recuperar la sencillez del lenguaje, no sólo para llegar con más facilidad al lector, sino también para desasirse de la perversión que el nazismo había operado con las palabras. Sus relatos, pues, comparten rasgos generacionales ya enumerados en el apartado i (acción cotidiana, ruptura de un destino, acción mínima, etc.).

Sin embargo, se destaca como una creadora muy original, distanciada de los cánones generacionales. El segundo objetivo de este estudio consistía en desgranar tal singularidad. Tras ofrecer una perspectiva general de su catálogo (apartado 2), nos centramos en lo que ella misma señaló como lo más genuino de su aportación literaria:

- Flexibilidad en el uso de las marcas formales (espacio, tiempo...) que definen el género.

- Pese a su fragmentariedad o finitud, concebir el relato como una unidad completa de sentido.

- La realidad nos aparece como algo "extraño" al tiempo que los personajes son dominados por fuerzas "opacas" para ellos mismos.

- Limitación del alcance de las explicaciones racionales. El miedo como estado fundamental frente a un entorno incontrolable.

Y lo que Pulver, Lenz y Reich-Ranicki han aportado en este sentido, centrándose sobre todo en el tema del destino, de cómo se rompe imprevisiblemente, lo que nos influye, y lo lejos que queda de nuestro alcance su control. 


\section{Referencias bibliográficas}

- Bienek, H. (I962). Werkstattgespräche mit Schrifstellern. München: Carl Hansel Verlag.

- Bönnighausen, M. \& Vogt, J. (20I3). Literatur für die Schule: Ein Handbuch (Vol. 8522). Stuttgart: UTB.

- Brustmeier, H. (i966). Der Durchbruch der Kurzgeschichte in Deutschland. Versuch einer Typologie der Kurzgeschichte, dargestellt am Werk Wolfgang Borcherts. Universität Marburg: Inauguraldissertation (autoedición).

- Freese, P. (1974). Die amerikanische Kurzgeschichte nach 1945 (Vol. 8). Frankfurt am Main: Athenäum.

- Grabert-Mulot-Nürnberger. (1982). Geschichte der deutschen Literatur. München: Bayerischer Schulbuch-Verlag.

• Hegel, G. W. F. (1973). Phänomenologie des Geistes. Frankfurt am Main: Suhrkamp.

- Kaschnitz, M. L. \& Büttrich, C. (I98I). Gesammelte Werke: in sieben Bänden. Frankfurt am Main: Insel-Verlag.

- Kuipers, J. (1970). Zeitlose Zeit: die Geschichte der deutschen Kurzgeschichtsforschung. Gronningen: Wolters-Noordhoff.

- Langenbucher, W. R., \& Silvetti Paz, N. (1970). Narradores alemanes contemporáneos. Buenos Aires: Editorial Sudamericana.

-Lenz, S. (I966, 2 I de noviembre), “Personen mit Schicksal”. Der Spiegel.

- Martín Arnedo, S. (2OI5). Das Unheimliche como clave hermenéutica en los relatos de ML Kaschnitz. Revista de Filología Alemana, 23, 77-95.

- Marx, L. (2005). Die deutsche Kurzgeschichte. Stuttgart: Springer-Verlag.

- Poe, E. A. (I846). The Philosophy of Composition. Graham 's American Monthly Magazine, vol. XXVIII, I63-I67.

- Pulver, E. (1984), Marie Luise Kaschnitz (Vol. 40), CH Beck.

- Reich-Ranicki, M. (I966, 23 de septiembre), “... die uns überleben werden”. Die Zeit. 
- Schnell, R. (200I), Deutsche Literatur nach 1945. Deutsche Literaturgeschichte, Stuttgart, J. B. Metzler.

- Schweikert, U. (I984). Marie Luise Kaschnitz. Frankfurt am Main: Suhrkamp Taschenbuch Materialien.

- Tapies, A. (1978). El arte contra la estética. Barcelona: Ariel.

- Titzmann, M. (1977). Strukturale Textanalyse: Theorie und Praxis der Interpretation, München: Fink.

-Weyrauch, W. (ı949). Tausend Gramm. Sammlung neuer deutscher Geschichten. Hamburg: Rowolth. 